\title{
Correction to: Clinical and Immunological Heterogeneity in Japanese Patients with Gain-of-Function Variants in STAT3
}

\author{
Kay Tanita ${ }^{1} \cdot$ Fumiaki Sakura ${ }^{2} \cdot$ Ryusuke Nambu $^{3,4,5} \cdot$ Miyuki Tsumura $^{2} \cdot$ Yusuke Imanaka $^{2} \cdot$ Hidenori Ohnishi $^{6}$. \\ Zenichiro Kato ${ }^{6,7} \cdot$ Jie Pan $^{4,5}$ - Akihiro Hoshino ${ }^{1} \cdot$ Koji Suzuki $^{8}$ - Motoko Yasutomi ${ }^{8}$. Shuichiro Umetsu ${ }^{9}$. \\ Chizuru Okada $^{10} \cdot$ Masatoshi Takagi $^{1} \cdot$ Kohsuke Imai $^{11}$ - Osamu Ohara ${ }^{12,13} \cdot$ Alexo M. Muise $^{4,5,14} \cdot$ Satoshi Okada $^{2}$. \\ Tomohiro Morio ${ }^{1} \cdot$ Hirokazu Kanegane ${ }^{15}$
}

Published online: 10 March 2021

(C) Springer Science+Business Media, LLC, part of Springer Nature 2021

\section{Correction to: Journal of Clinical Immunology 2021 https://doi.org/10.1007/s10875-021-00975-y}

After the publication of the original article, we noticed to need corrections in Figures. In Fig. 1a, closed square in patient 3 is changed to closed circle, and closed circle in patient 5.1 is changed to closed square. IL-6 is changed to FP-6 in Fig. 2b, and FP-6 is added in Fig. 2c. In Fig. 4c, an extra IL6 is deleted. Please see below corrected version. We apologize for any inconvenience that this may have caused.

Kay Tanita, Fumiaki Sakura and Ryusuke Nambu contributed equally to this work.

The online version of the original article can be found at https://doi.org/ 10.1007/s10875-021-00975-y

Hirokazu Kanegane

hkanegane.ped@tmd.ac.jp

1 Department of Pediatrics and Developmental Biology, Graduate School of Medical and Dental Sciences, Tokyo Medical and Dental University (TMDU), Tokyo, Japan

2 Department of Pediatrics, Hiroshima University Graduate School of Biomedical and Health Sciences, Hiroshima, Japan

3 Division of Gastroenterology and Hepatology, Saitama Children's Medical Center, Saitama, Japan

4 SickKids Inflammatory Bowel Disease Center, The Hospital for Sick Children, Toronto, ON, Canada

5 Cell Biology Program, Research Institute, The Hospital for Sick Children, Toronto, ON, Canada

6 Department of Pediatrics, Gifu University Graduate School of Medicine, Gifu, Japan

7 Structural Medicine, United Graduate School of Drug Discovery and Medical Information Sciences, Gifu University, Gifu, Japan
8 Department of Pediatrics, Faculty of Medical Sciences, University of Fukui, Fukui, Japan

9 Department of Pediatric Hepatology and Gastroenterology, Saiseikai Yokohama-shi Tobu Hospital, Yokohama, Kanagawa, Japan

10 Hiroshima Chuodori Children Clinic, Hiroshima, Japan

11 Department of Community Pediatrics, Perinatal and Maternal Medicine, Graduate School of Medical and Dental Sciences, Tokyo Medical and Dental University (TMDU), Tokyo, Japan

12 Department of Technology Development, Kazusa DNA Research Institute, Chiba, Japan

13 Laboratory for Integrative Genomics, RIKEN Center for Integrative Medical Sciences, Yokohama, Japan

14 Department of Pediatrics, Institute of Medical Science and Biochemistry, University of Toronto, the Hospital for Sick Children, Toronto, ON, Canada

15 Department of Child Health and Development, Graduate School of Medical and Dental Sciences, Tokyo Medical and Dental University (TMDU), 1-5-45 Yushima, Bunkyo-ku, Tokyo 113-8519, Japan 
a
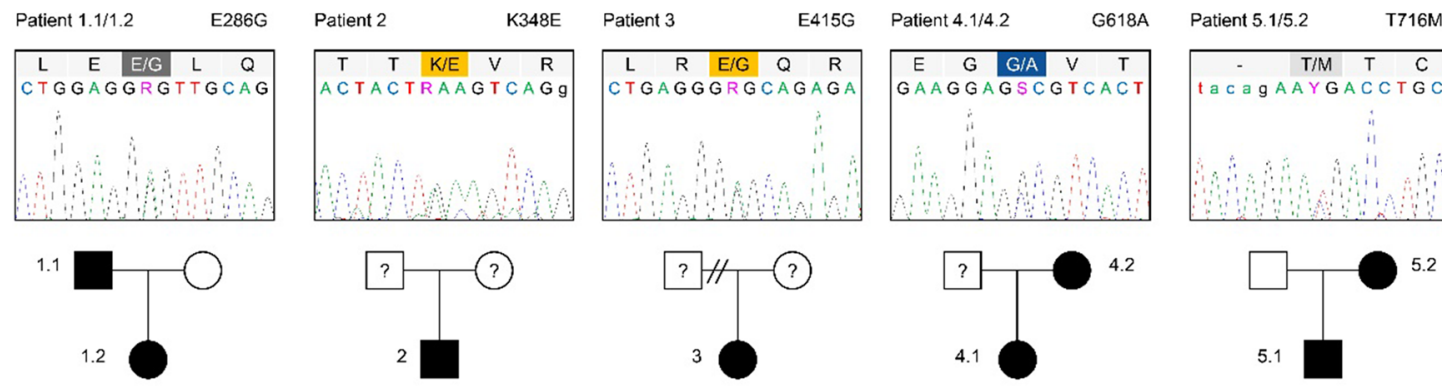

b

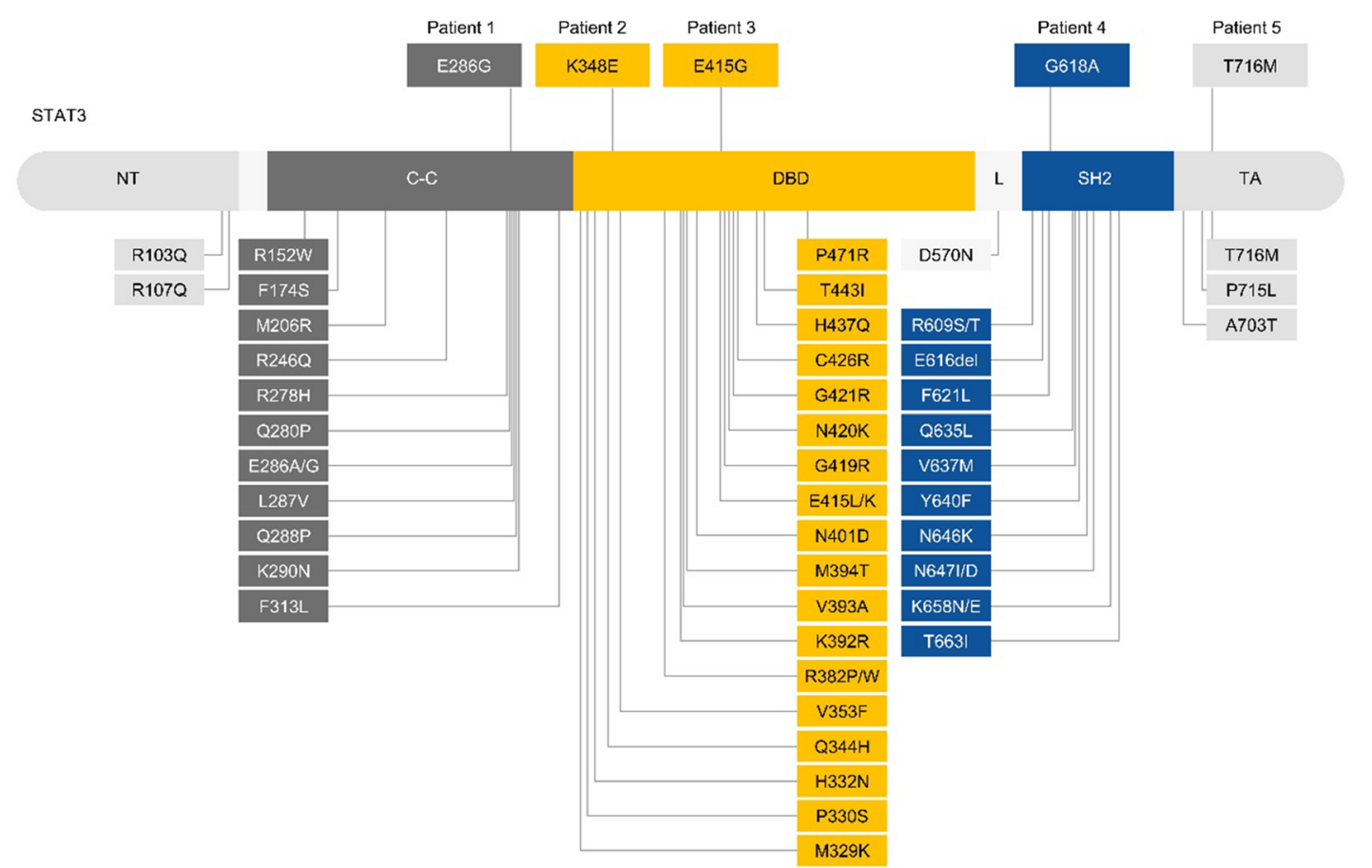

Fig. 1 Sanger sequencing of STAT3, (a) Family tree and Sanger sequencing results for each patient family. (b) Schematic of the STAT3 protein showing the location of the variants and the resulting amino acid changes. Upper panel indicates the variants identified in this study, whereas lower panel indicates previously reported pathogenic variants of STAT3-GOF. STAT3 is divided into six domains: N-terminal (NT), coiled-coil (C-C), DNA binding (DBD), linker (L), Src-homology 2 (SH2), and transactivation (TA) 
Fig. 2 Reporter assay and western blot of STAT3, (a) STAT3 reporter activity in the presence or absence of IFN- $\alpha$, FP6, and IL-27. E415K and R382W are known GOF and LOF mutants, respectively. Asterisks and double asterisks indicate $p<$ 0.05 and $p<0.01$, respectively. (b) Western blot analysis of transfected A4 cells after stimulation with FP6. (c) Quantification of protein band intensity (pSTAT3/STAT3) was quantified using ImageJ software (National Institutes of Health, Bethesda, MD) a

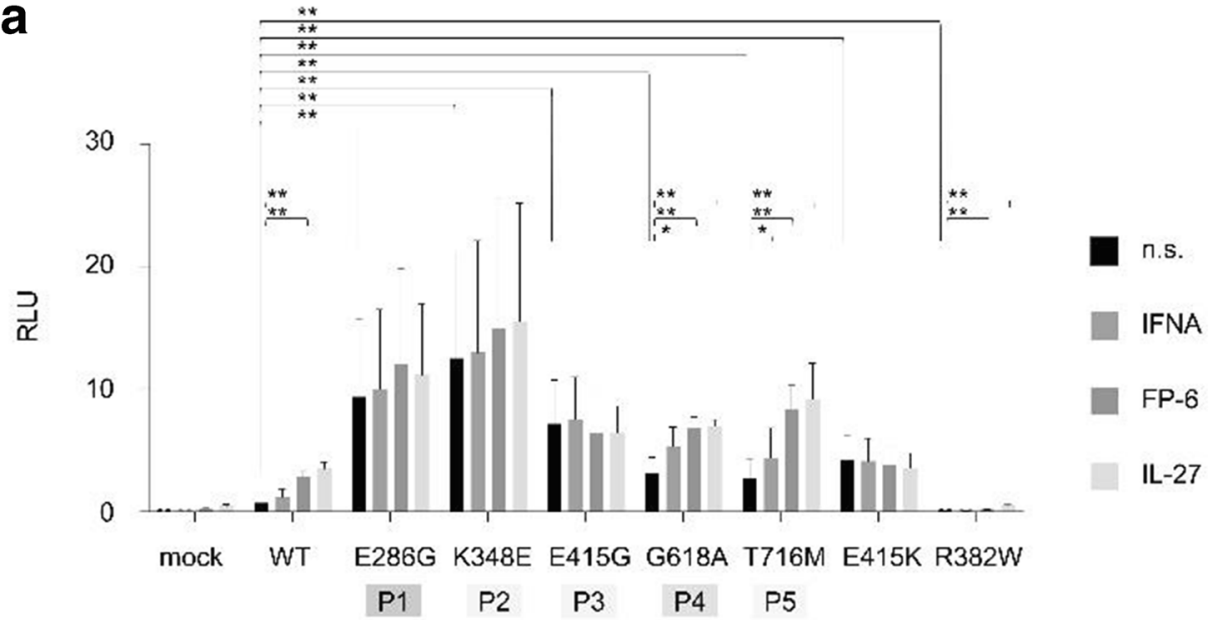

b
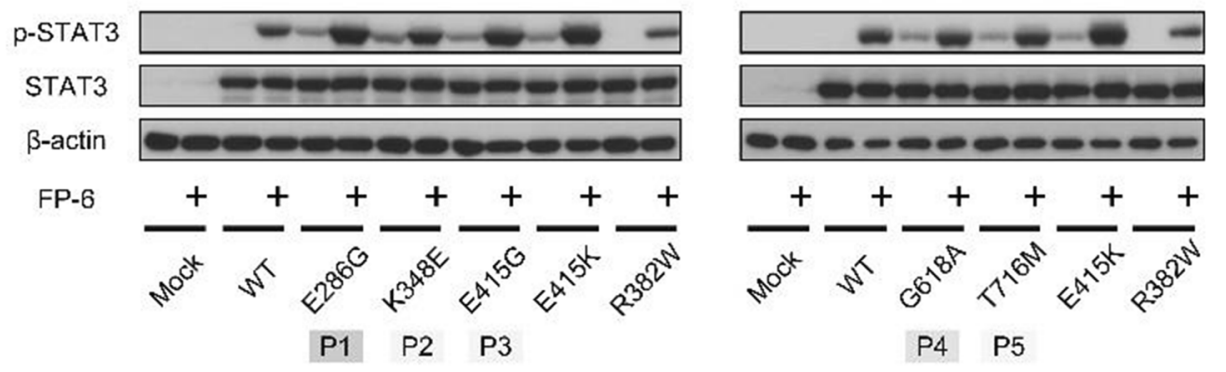

C

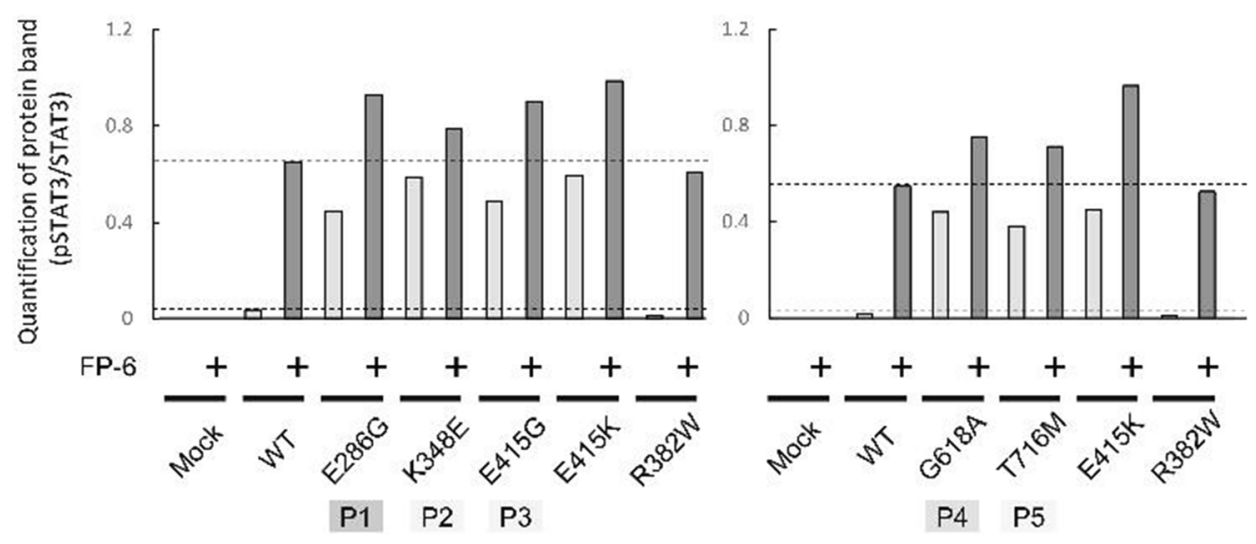




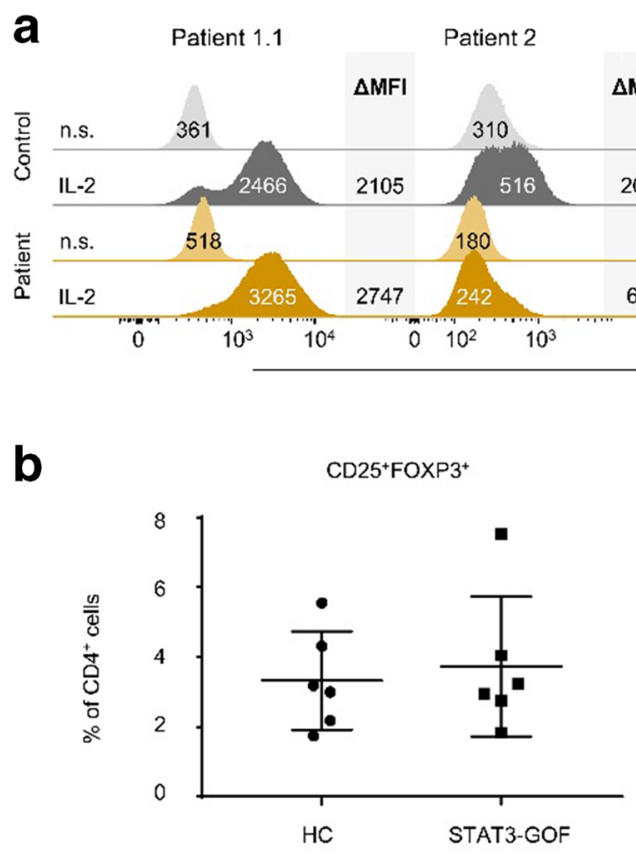

d

Patient 3

Patient 4.1

Patient 4.2

Patient 5.1

$\triangle M F I$

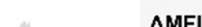

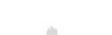

$\triangle M F I$
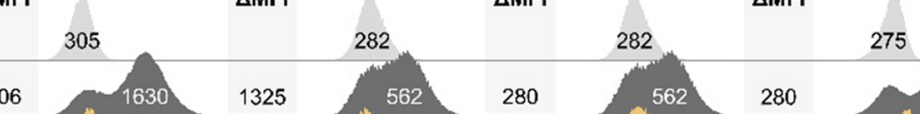

$\triangle M F I$

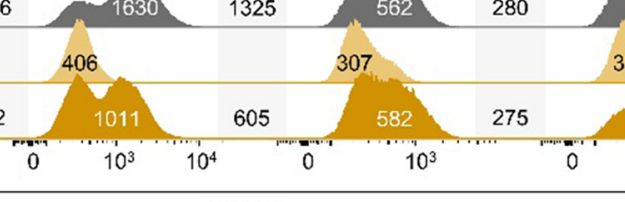

pSTATS

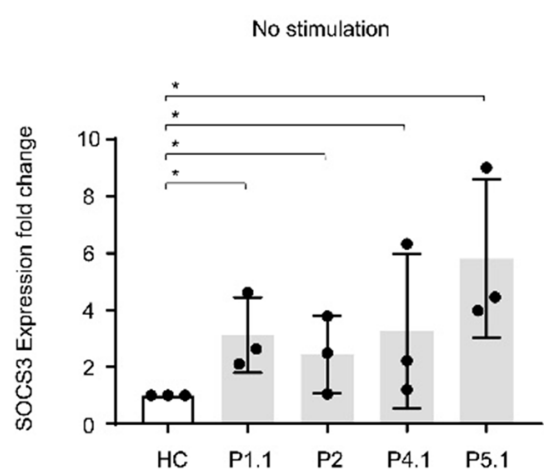

b

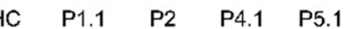

C

FOXP3+CD127

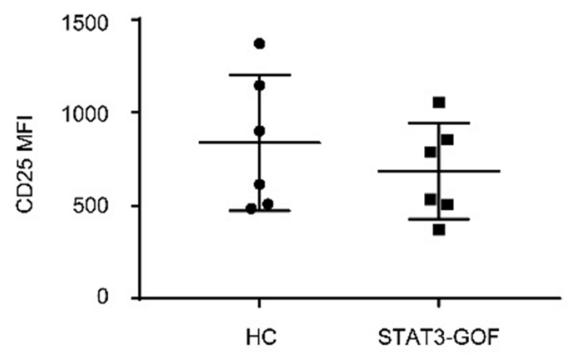

IL-6 stimulation $(50 \mathrm{ng} / \mathrm{mL})$

IL-21 stimulation (50 ng/mL)

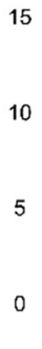

$10]$
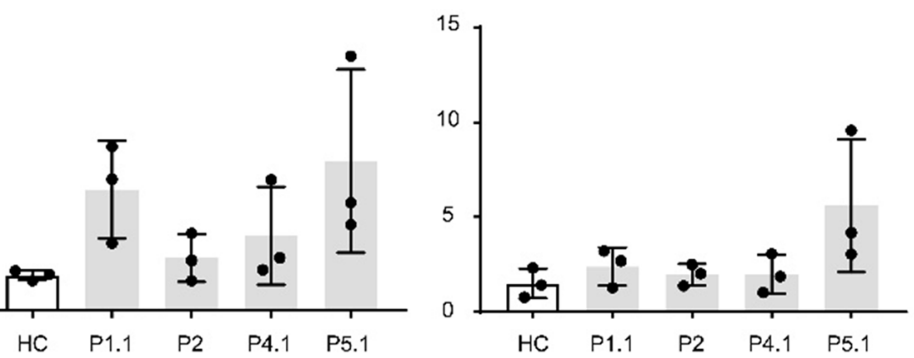

Fig. 4 Immunological analysis of patient PBMCs, (a) Flow cytometric analysis of intracellular phosphorylated STAT5 in primary PBMCs after IL-2 stimulation. MFI, mean fluorescence intensity. (b) Percentage of patients. (c) MFI of $\mathrm{CD} 25$ in $\mathrm{FOXP} 3{ }^{+} \mathrm{CD} 127^{\text {dim }}$ cells. (d) Real-time PCR analysis of SOCS3 in patient-derived EBV-LCLs, in the presence or absence of IL-6 and IL-21 stimulation. Asterisks indicate $p<0.05$

Publisher's Note Springer Nature remains neutral with regard to jurisdictional claims in published maps and institutional affiliations. 